\title{
Actinomicosis pélvica. A propósito de un caso que simula un tumor de recto
}

\author{
Javier Faúndez S. ${ }^{1}$, Sebastián A. Uribe A. ${ }^{1}$ y Felipe S. Pizarro C. ${ }^{1}$
}

\section{Pelvic actinomycosis. From a case that simulates rectal tumor}

Introduction: Actinomycosis is a rare chronic infection caused by bacterias of the genus Actinomyces sp. Pelvic involvement is one of its most infrequent forms and in many cases it is related to the use of a longstanding intrauterine device or a previous surgery. Like other rare diseases, the infection is known as "the great imitator" because of its varied form of presentation and its particular behavior, which can simulate a neoplasm. The treatment is fundamentally medical with good results. Case Report: We present the case of a patient who presented with a rectal tumor but that turned out to be Actinomycosis. The diagnosis was made based on the Gram stain, the clinical presentation and the history of an intrauterine device left for more than 25 years. It was subsequently corroborated by pathological anatomy and successfully treated with antibiotics for an extended period. Conclusion: Although actinomycosis is an infrequent pathology, it should be considered in the differential diagnosis of patients who present with tumors of the pelvis. An active diagnostic attitude and a high index of suspicion are fundamental for the timely, safe and effective treatment of this disease.

Key words: actinomicosis; pelvic infection; rectal tumor; colonoscopy; IUD.

\section{Resumen}

Introducción: La actinomicosis, es una infección crónica rara producida por bacterias del género Actinomyces sp. La afectación pélvica es una de sus formas más infrecuentes y en gran parte de los casos se relaciona al uso de un dispositivo intrauterino de larga data o a una cirugía previa. Como otras enfermedades raras, la infección es conocida como "la gran imitadora" por su variada forma de presentación y particular comportamiento pudiendo simular una neoplasia. El tratamiento es fundamentalmente médico y de buenos resultados. Caso Clínico: Damos a conocer el caso de una paciente que se presentó con un cuadro compatible con un tumor de recto, pero que resultó ser actinomicosis. El diagnóstico se realizó en base a la tinción de Gram, el cuadro clínico y el antecedente de un dispositivo intrauterino abandonado por más de 25 años. Fue corroborado posteriormente mediante anatomía patológica y tratada en forma exitosa con antibióticos por un periodo extendido. Conclusión: Si bien la actinomicosis es una patología infrecuente, debe ser considerada en el diagnóstico diferencial de los pacientes que se presentan con tumores de la pelvis. Un alto índice de sospecha y una actitud diagnóstica activa son fundamentales para un tratamiento oportuno, seguro y eficaz de esta enfermedad.

Palabras clave: actinomicosis; infección pélvica; tumor de recto; colonoscopía; DIU.

\section{Introducción}

La actinomicosis es una infección crónica e infrecuente que tiende a formar granulomas abscedados de crecimiento lentamente progresivos simulando otras condiciones como neoplasias o tuberculosis. El agente causal es una bacteria del género Actinomyces sp; y corresponden a bacilos largos Gram positivos, anaeróbicos, no esporulados, que colonizan normalmente la orofaringe, tracto gastrointestinal y tracto urogenital femenino. La enfermedad ocurre típicamente al existir una brecha en la mucosa manifestándose en la región cervicofacial (50\%), torácica (15-20\%), abdomino-pélvica $(20 \%)$ y otras formas excepcionales como en el $\mathrm{SNC}^{1,2}$.

Se describen diferentes subtipos que pueden
${ }^{1}$ Hospital de la Fuerza Aérea

de Chile.

Santiago, Chile.

Recibido el 17 de octubre de 2018 y aceptado para publicación el 14 de diciembre de 2018.

Correspondencia a Dr. Sebastián Uribe A sebastian.uribe@gmail.com 
producir la enfermedad en el ser humano, siendo en la mayoría de los casos Actinomyces israelii. Otras cepas corresponden a $A$. naeslundii, A. odontolyticus, A. viscosus y A. meyeri ${ }^{3,4}$.

La infección puede ocurrir a todas las edades sin embargo, se presenta principalmente entre la cuarta y quinta década de la vida. Por lo general hay una mayor prevalencia en el sexo masculino (hasta 3 veces más), con excepción de la actinomicosis pélvica que afecta principalmente a mujeres, y especialmente a aquellas que son usuarias de dispositivos intrauterinos (DIU) $)^{1,2,5}$.

El diagnóstico por cultivo, si bien es específico, es positivo en apenas $30-50 \%$ de los casos, siendo más habitual encontrar un organismo gram $(+)$ y la presencia de los característicos "gránulos azurófilos" (conglomerados de actinomicetos) en muestras de anatomía patológica, que si bien apoyan fuertemente el diagnóstico, no son patognomónicos y se logran identificar en solo la mitad de los $\operatorname{casos}^{5,6-10}$. El tratamiento se realiza en base a antibióticos derivados de la penicilina y tradicionalmente se ha recomendado por un período prolongado; sin embargo, en la actualidad, se recomienda que la duración del tratamiento sea evaluado caso a caso. Para pacientes alérgicos, eritromicina, clindamicina o tetraciclina son buenas opciones terapéuticas ${ }^{1,5}$.

La actinomicosis pélvica es una de las presentaciones más infrecuentes existiendo pocos reportes en la literatura, especialmente a nivel nacional ${ }^{3,7,8}$. En este contexto, presentamos el caso clínico de una mujer con una infección pélvica que progresó por los parametrios invadiendo los espacios preperitoneal y pelvirectal, expresándose morfológicamente como un tumor rectal y posteriormente como un absceso de la pared abdominal, hecho que permitió oportunamente su diagnóstico.

\section{Caso Clínico}

Paciente femenino de 60 años, hipertensa en tratamiento, consulta de manera ambulatoria por dolor insidiosamente progresivo en hemiabdomen inferior de aproximadamente 40 días de evolución. No presentaba otros síntomas asociados como fiebre, escalofríos, baja de peso o sangrado anal. Al examen físico destacaba una mayor resistencia muscular hacia la FII.

Se solicitó estudio con tomografía computada de abdomen y pelvis con contraste que evidenció un engrosamiento mural del recto distal con aumento de la densidad del tejido adiposo perirectal (Figura 1) y colonoscopía que mostró una lesión a $10 \mathrm{~cm}$ del margen anal, no ulcerada, elevada y estenosante (tipo Bormann II) y cuyas biopsias demostraron cambios inflamatorios inespecíficos (Figura 2).

Cuatro semanas más tarde acude por aumento del dolor abdominal observándose una masa en el cuadrante inferior izquierdo del abdomen, sensible y de consistencia firme, el que primeramente se interpretó como una hernia de la línea semilunar atascada por lo que se hospitalizó de urgencia. La paciente no presentaba signos de obstrucción intestinal y la tomografía realizada al ingreso demostró una masa de centro líquido, con progresión de los cambios inflamatorios rectales respecto a control previo el que fue informado como una enfermedad neoplásica vs inflamatoria (Figuras 3 y 4).

Se decidió manejo quirúrgico y se encontró un absceso que provenía del espacio preperitoneal, rompía la aponeurosis abdominal y se coleccionaba en el espacio subcutáneo. La tinción de Gram del

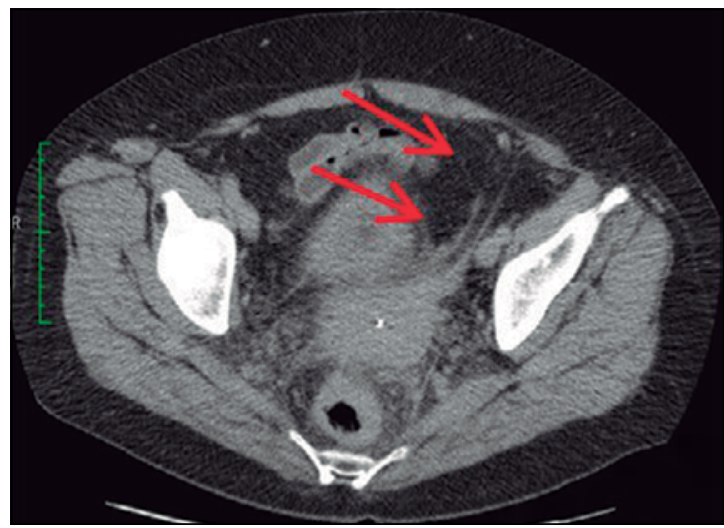

Figura 1. Tomografía computada. Engrosamiento mural de recto. Nótese como progresa la inflamación a través del mesometrio (flechas).

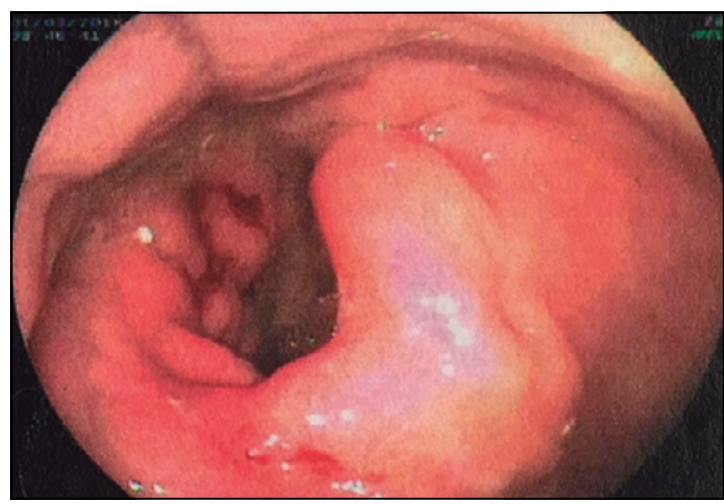

Figura 2. Colonoscopía. Lesión solevantada en rodete ocupando aproximadamente la mitad de la circunferencia. (Tipo II de Bormann). 


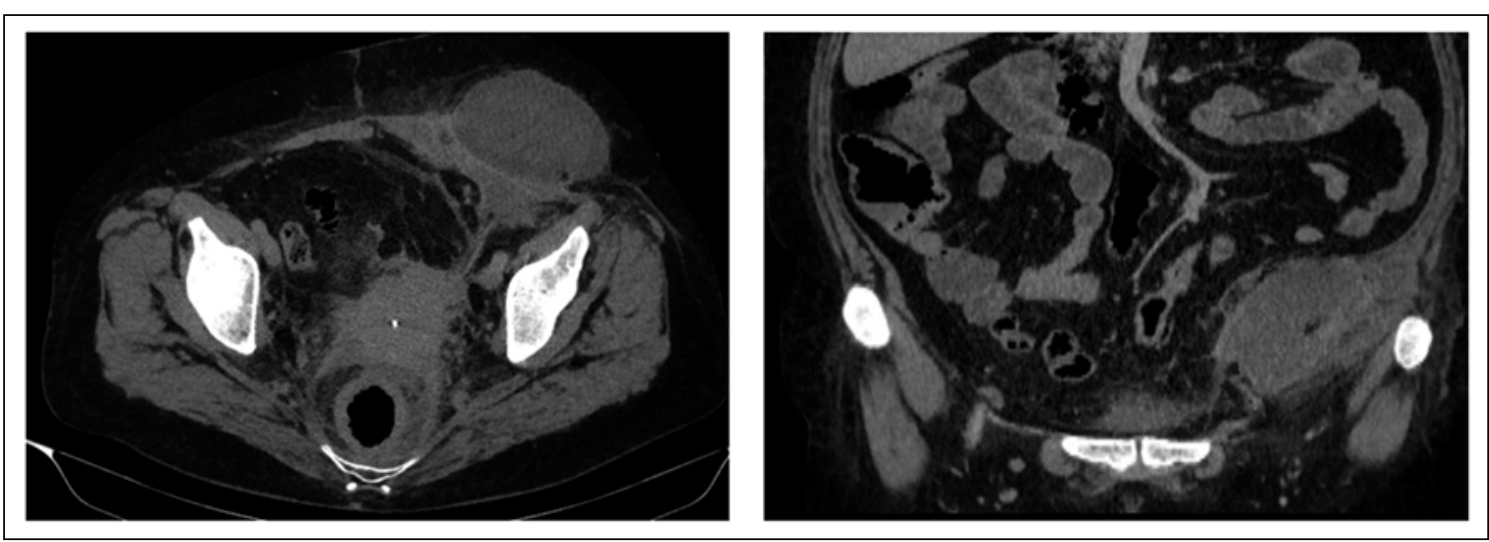

pus obtenido mostró escasos bacilos $\mathrm{G}(+)$ largos, lo que unido al cuadro clínico abigarrado y la presencia de un dispositivo intrauterino abandonado por más de 25 años hicieron sospechar el diagnóstico. Los cultivos obtenidos con posterioridad resultaron negativos, tanto para gérmenes aerobios como anaerobios. Las baciloscopías también resultaron negativas. El análisis histológico -solicitado dirigidamente- mostró una lesión supurada con gránulos azurófilos apoyando el diagnóstico (Figura 5). Desde el postoperatorio inmediato la paciente fue tratada con penicilina y posteriormente con amoxicilina/Ac. clavulánico durante 3 meses presentando una evolución favorable.

\section{Discusión}

La actinomicosis es una enfermedad poco frecuente y de difícil diagnóstico debido principalmente a su clínica inespecífica y a la vez al ser "imitadora" de otras patologías tales como neoplasias, infecciones por tuberculosis, diverticulitis, absceso tubo-ovárico, Crohn entre otras, haciendo que el diagnóstico al ingreso sea sospechado en apenas el $8 \%$ a $17 \%$ de los $\operatorname{casos}^{5,11}$.

Los pacientes, en su mayoría del sexo femenino, (actinomicosis ginecológica), consultan mayoritariamente por dolor pélvico crónico, baja de peso, tumor palpable o metrorragia ${ }^{9-20}$; existiendo, tal como en nuestro caso, una predisposición documentada con el uso de un dispositivo intrauterino. Lo anterior es perfectamente observable en el trabajo nacional de Madrid y cols., en que $81,2 \%$ de las pacientes estudiadas con actinomicosis resultaron ser usuarias de este método anticonceptivo y en la revisión de Torres y col, en que de un total de 1.774 informes de PAPs de una comunidad rural de Chile, con 15 resultados positivos para actinomyces, el $86,7 \%$ de las pacientes eran portadoras de DIU ${ }^{4,9}$. Otros reportes han comunicado la presencia de actinomices en frotis cervicales en usuarias de DIU un promedio de $7 \%$, con rangos tan variables de entre 0 a $31 \%$, sin embargo, estos rangos estarían más probablemente dados por falsos positivos y si bien no se ha logrado establecer el riesgo a desarrollar enfermedad entre usuarias de DIU, se ha visto que la infección puede provocar infertilidad e incluso mortalidad; por

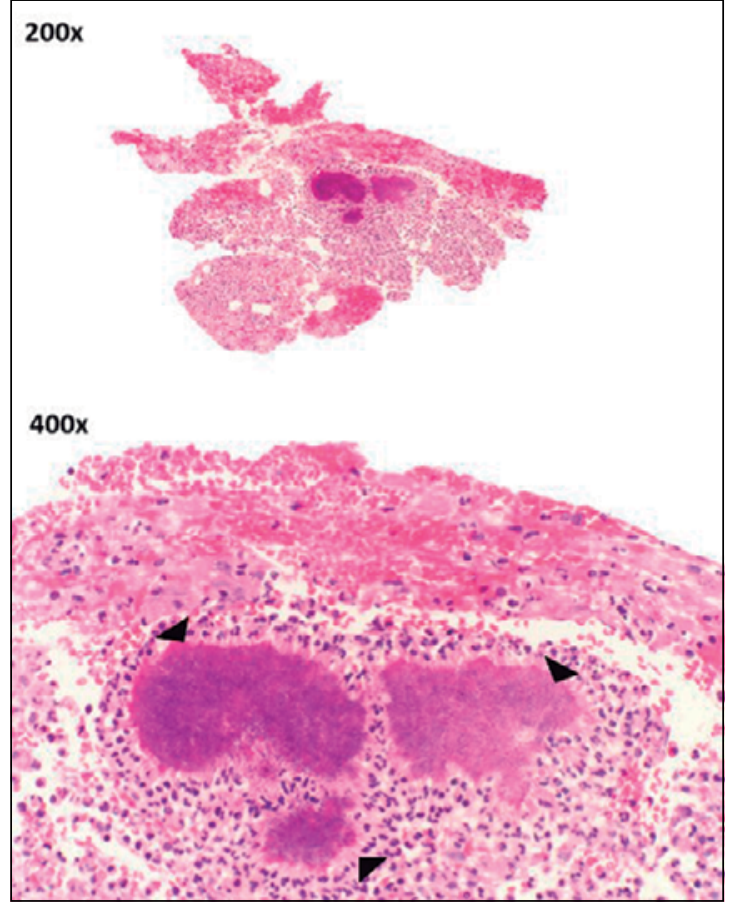

Figura 5. Microfotografía de los bordes del absceso. Hematoxilina-Eosina (200x y 400x). Se visualizan los característicos "gránulos azurófilos" (flechas).
3 y 4 . Tomografía computada. Corte axial y coronal respectivamente. semanas después. Se observa progresión de la inflamación con formación quido de $11 \times 11 \times 10 \mathrm{cms}$ 
lo tanto, parece sensato que frente a un resultado positivo de PAP para actinomyces, el retiro de DIU sea recomendado ${ }^{9,13}$. Es importante recalcar que en Chile el examen de citología cervical exfoliativa de papanicolau es parte del Programa Nacional de Prevención del Cáncer Cérvico-Uterino desde 1987.

Pensamos que para el caso clínico expuesto, el DIU al actuar como cuerpo extraño de la cavidad uterina, facilitó el crecimiento y diseminación del actinomyces a través de los parametrios, alcanzando así la pared pélvica izquierda y el tejido adiposo perirectal posterior y lateral, lo que produjo la imagen de tumor vista en la colonoscopia. Consideramos que lo expuesto sin duda es novedoso, pues a nuestro mejor entender, es la primera vez que se reporta en español una lesión que simula un tumor de recto tipo Bormann II donde característicamente no había afectación de la mucosa, tal como en otros casos de la literatura ${ }^{11,12,14,15}$.

Respecto al estudio imagenológico podemos señalar que la elección es la tomografía computada pues permite estimar la ubicación y extensión de la infección poniendo en evidencia la naturaleza infiltrativa de la enfermedad que invade a través de los planos y límites anatómicos de los órganos involucrados $^{10}$. No obstante lo anterior, los hallazgos a la tomografía son inespecíficos, lo que hace plantear el diagnóstico diferencial con absceso tubo-ovárico, enfermedad de Crohn, diverticulitis, endometriosis, cáncer de colon, cáncer de ovario, cáncer de próstata, entre otros ${ }^{16}$. Dentro de los hallazgos, lo más habitual es ver un engrosamiento parietal concéntrico asociado a una masa quística, sólido-quística y más rara vez puramente sólida. Otros hallazgos incluyen tejido graso perilesional de aspecto heterogéneo y nula o mínima linfoadenopatia ${ }^{17,18}$. Los órganos más afectados son la región ileocecal y el intestino delgado para la actinomicosis abdominal y los ovarios, trompas y rectosigmoides para la actinomicosis pélvica $^{10,19}$.

El tratamiento es principalmente de tipo médico basado en el uso de antibióticos; sin embargo, en la mayoría de los casos existe una cirugía previa ya sea por las dificultades que acarrea el diagnóstico preoperatorio, a la presencia de signos peritoneales o como en esta paciente a la existencia de gran- des cavidades abscedadas que hacen necesario el drenaje quirúrgico o percutáneo ${ }^{5-9}$. Clásicamente el tratamiento de elección ha sido penicilina $\mathrm{y}$ sus derivados en dosis altas por un periodo largo de 6 a 12 meses; sin embargo, el enfoque actual es un tratamiento individualizado que dependerá de la respuesta clínico-radiológica del paciente, existiendo reportes exitosos con apenas 2 meses de tratamiento. En una experiencia internacional de 23 pacientes con actinomicosis pélvica a lo largo de 15 años se vio que ninguno presentó recurrencia luego de un seguimiento promedio de 30 meses lo que nos habla de la buena respuesta al tratamiento ${ }^{5}$; no obstante, lo anterior la enfermedad puede resultar bastante agresiva reportándose importante morbilidad e incluso mortalidad. Los reportes respecto a esta última son variables y dependerán del sitio involucrado en la infección, de las condiciones del paciente y de la precocidad con que se instaure el tratamiento $^{1,20}$.

\section{Conclusiones}

La actinomicosis es un cuadro clínico de difícil diagnóstico; aunque infrecuente, la infección debe ser considerada dentro del diagnóstico diferencial de los tumores pélvicos, especialmente en mujeres.

Dado que el pilar del tratamiento es principalmente médico y de buenos resultados terapéuticos, es fundamental un alto índice de sospecha junto a una actitud diagnóstica activa, de manera de evitar tratamientos que sean innecesarios o riesgosos, especialmente si se confunde con una neoplasia.

\section{Responsabilidades éticas}

Protección de personas y animales. Los autores declaran que para esta investigación no se han realizado experimentos en seres humanos ni en animales.

Confidencialidad de los datos. Los autores declaran que en este artículo no aparecen datos de pacientes.

Conflictos de interés: no hay. 


\section{Bibliografía}

1. Wong VK, Turmezei TD, Weston VC. Actinomycosis. BMJ 2011;343:d6099.

2. Bonnefond S, Catroux M, Melenotte C, Karkowski L, Rolland L, Trouillier S, et al. Clinical features of actinomycosis: A retrospective, multicenter study of 28 cases of miscellaneous presentations. Medicine (Baltimore). 2016;95:e3923.

3. Alegría BJ, González MMP, Galleguillos CM, Whittle PC, Franco SC. Revisión de infección pelviana por actinomices: presentación de un caso clínico. Revista Chilena de Radiología. Sociedad Chilena de Radiología 2003;9:196-200.

4. Torres GS, Schalper CK. Análisis de la presencia de actinomicosis pélvica en mujeres de una comunidad rural en Chile. Rev Chil Obstet Ginecol. 2002;67:232-6.

5. Sung HY, Lee IS, Kim SI, Jung SE, Kim SW, Kim SY, et al. Clinical features of abdominal actinomycosis: a 15 -year experience of a single institute. J Korean Med Sci. 2011;26:932-7.

6. Cruz Choappa R, Vieille Oyarzo P. Histological diagnosis of actinomycosis. Rev Argent Microbiol. 2018;50:108-10.

7. Daniels SP, Correa AE, Goity FC. Actinomicosis pelviana: caso clínico. Rev Chil Obstet Ginecol. 2013;78:48-50.
8. Bannura G, Contreras J, Portalier P. Actinomicosis colorrectal. Rev Chil Cir. 1995;47:622-7.

9. Madrid S. Freddy, Díaz Z. Sergio, Mucientes Francisco, Klaassen Rodrigo. Actinomicosis ginecologica. Rev. Chil. Obstet. Ginecol. [Internet]. 2003 [citado 2018 Jul 13] ;68: 21-27.

10. Bae JH, Song R, Lee A, Park J-S, Kim MR. Computed tomography for the preoperative diagnosis of pelvic actinomycosis. J Obstet Gynaecol Res. 2011;37:300-4.

11. Triantopoulou C, Van der Molen A, Van Es AC, Giannila M. Abdominopelvic actinomycosis: spectrum of imaging findings and common mimickers. Acta Radiol Short Rep. 2014 Feb 21;3(2):2047981614524570. doi: 10.1177/2047981614524570. eCollection $2014 \mathrm{Feb}$.

12. Ji W, Kwak JM, Kim J, Kim SH. Actinomycosis of the rectum mimicking a malignant neoplasm. ANZ J Surg. 2014;84:497.

13. Westhoff C. IUDs and colonization or infection with Actinomyces. Contraception. 2007;75(6 Suppl):S48-50.

14. Petersen $\mathrm{S}$, Würschmidt $\mathrm{F}$, Gaul H, Caselitz J, Schwenk W. Actinomyces infection mimicking tumor-progression in rectal cancer under neoadjuvant therapy. Acta Oncol. 2012;51:270-2.

15. Dumitru E, Dumitru IM, Popescu

R, Resul G, Bulbuc I, Rugina S Simultaneous occurrence of two rare diseases: actinomycosis and melanoma of the rectum. J Gastrointestin Liver Dis. 2014;23:95-8.

16. Garner JP, Macdonald M, Kumar PK. Abdominal actinomycosis. Int J Surg. 2007;5:441-8.

17. Alothman M. Abdominopelvic Actinomycosis: Radiographic Features. Clin Radiol Imaging J. 2018;2:000114.

18. Lee IJ, Ha HK, Park CM, Kim JK, Kim $\mathrm{JH}, \mathrm{Kim} \mathrm{TK}$, et al. Abdominopelvic actinomycosis involving the gastrointestinal tract: $\mathrm{CT}$ features. Radiology 2001;220:76-80.

19. Heo SH, Shin SS, Kim JW, Lim HS, Seon HJ, Jung S-I, et al. Imaging of actinomycosis in various organs: a comprehensive review. Radiographics 2014;34:19-33.

20. Nozawa H, Yamada Y, Muto Y, Arita S, Aisaka K. Pelvic actinomycosis presenting with a large abscess and bowel stenosis with marked response to conservative treatment: a case report. J Med Case Rep. 2007;1:141. 